\title{
REFLEXIONES SOBRE EL DERECHO FRENTE A LAS TECNOLOGÍAS DISRUPTIVAS
}

\author{
Margarita Robles Carrillo \\ Profesora Titular de Derecho Internacional Público \\ Universidad de Granada, España
}

\begin{abstract}
RESUMEN
La evolución de las tecnologías de la información y la comunicación (TIC) constituye un desafío para el Derecho, en particular, con las llamadas tecnologías disruptivas entre las que destaca la inteligencia artificial (IA). El debate sobre la IA se ha centrado en sus aspectos tecnológicos y éticos, mientras que se ha infravalorado o soslayado la función del Derecho. La necesidad de proceder a la regulación de la IA se ha puesto de manifiesto en el marco institucional y doctrinal. El estudio de la práctica muestra que son muy pocos los Estados que ha adoptado medidas nacionales y, en el marco internacional, la mayoría de las iniciativas están localizadas en organizaciones regionales en las que participan los países tecnológicamente desarrollados. Esta situación no significa que exista un vacío legal. El Derecho internacional tiene mecanismos de respuesta que permitan configurar el marco normativo necesario para la gobernanza de la IA.
\end{abstract}

\section{PALABRAS CLAVE}

Inteligencia Artificial, Gobernanza, Estrategias, Derecho Internacional

\section{INTRODUCCIÓN}

La evolución de las tecnologías de la información y la comunicación (TIC) constituye un desafío para la humanidad en su conjunto y, académicamente, en particular, para las disciplinas filosóficas o científicas que se dedican al estudio de la sociedad y de los modelos de comportamiento diseñados para su organización y funcionamiento. Mientras que, técnicamente, el desarrollo se produce en progresión geométrica, en esas otras esferas de conocimiento, ni siquiera se alcanza un ritmo aritmético. No es extraño que se haya recurrido a la expresión "tecnologías disruptivas" para calificar estos avances de las TIC. Entre ellos que destaca, por su alcance y potencial, la inteligencia artificial (IA), razón por la cual es el objeto principal de este trabajo.

La IA es una tecnología que se compara con la inteligencia humana o, incluso, aspira a mejorarla o superarla. La denominada IA general (IAG), la super-inteligencia (Castel y Castel, 2016) o la singularidad sirven para designar algo más que objetivos tecnológicos. Desde hace tiempo, la idea de una IA ha alimentado utopías y distopías. Desde una perspectiva científica, plantea principalmente tres órdenes de problemas. En primer lugar, no existe una definición generalmente aceptada de IA (Scherer, 2016: 359-362), ni siquiera el concepto mismo está exento de polémica. En segundo término, a causa o como consecuencia de ello, se parte con frecuencia de dos presupuestos erróneos: por una parte, una visión antropomórfica de la IA, generalizada y alimentada especialmente por la literatura y los medios de comunicación, que no se corresponde con la realidad; y, por otra, una concepción unitaria de la IA que tampoco es real porque no todos los artificios, procesos o programas incluidos dentro de esa categoría genérica constituyen algo más que un componente mecánico que se encuentra lejos de merecer la calificación de inteligente. En tercer lugar, el estudio de la doctrina científica pone de manifiesto que, a pesar del reconocimiento del alcance global y de la naturaleza trasversal de la AI, el debate se ha centrado en sus aspectos tecnológicos y éticos, mientras que se ha infravalorado o soslayado la necesidad de abordar el discurso jurídico (Wagner, 2018: 1).

El estudio de la IA plantea problemas conceptuales, funcionales, analíticos, prácticos y, también, jurídicos. El objetivo de este trabajo es justificar la necesidad y la importancia de articular el discurso jurídico sobre la IA, identificando y relocalizando los aspectos principales de ese debate (Sección 2.1), considerando la situación práctica (Sección 2.2) y planteando la metodología jurídica de respuesta al problema de la regulación de la IA (Sección 2.3). 


\section{EL DISCURSO JURÍDICO}

La gobernanza de la IA está siendo objeto de un amplio y valioso debate en el que se advierte un problema principal: no hay un lenguaje común, ni una metodología única o realmente interdisciplinar. Al protagonismo lógico de sus aspectos tecnológicos se ha sumado una invocación generalizada, abrumadora por sus aportaciones y, en ocasiones, errónea de su dimensión ética. El error resulta no solo de la confusión entre lo ético y lo jurídico sino, sobre todo y es lo más preocupante, de una percepción sesgada de la ética.

\subsection{El Debate sobre la IA}

La tecnología (a) y la ética (b) están protagonizando el debate sobre la IA situándolo en un marco científico y metodológico simplificador respecto del objeto de estudio y excluyente para otras áreas de conocimiento como el derecho (c).

a) En el primer caso, la situación parece lógica en la medida en que la IA es una creación tecnológica, pero hay que valorarla con cautela. En la doctrina ya se está advirtiendo sobre los peligros de sustituir la "governance of technology" por una "governance by technology" (DeNardis y Musiani, 2014). Benvenisti reflexiona críticamente sobre una "Governance by machines" (Benvenisti, 2018:56). Danaher utiliza el término "algocracy" para designar "a threat to the legitimacy of public decision-making processes, which is posed by opacity of certain algocratic governance systems" (Danaher, 2016). Habermas utiliza, asimismo, el discurso de la legitimidad para cuestionar esta situación (Habermas, 1986). En definitiva, la aproximación tecnológica es incompleta y, en consecuencia, insuficiente. La sacralización de la tecnología puede convertir los medios en fines y revertir las prioridades, en particular, la construcción histórica de un orden social basado en criterios de legitimidad democrática.

b) El debate ético sobre la IA es muy necesario, pero está resultando deficitario desde una perspectiva cuantitativa, cualitativa, funcional y teleológica. Por una parte, la acumulación creciente de propuestas éticas, prácticamente inabarcables, no permite separar fácilmente lo importante de lo accesorio, ni facilita un diálogo transparente y con conocimiento. Más no es necesariamente mejor. Por otra parte, la calidad de las propuestas es globalmente discutible por dos motivos: 1) La autoría de la inmensa mayoría de ellas corresponde a actores públicos o privados situados en Estados tecnológicamente desarrollados que están debatiendo sobre IA, mientras que apenas existen aportaciones de la mayoría de los países, carentes de esa tecnología; y 2) Las propuestas responden mayoritariamente a la idea de una ética única o universal o de unos valores homogéneos comunes igualmente válidos para todos las personas, sociedades o civilizaciones cuando no existe una ética universal, aunque algunos de sus contenidos puedan ser generales o ampliamente compartidos (Castel y Castel, 2016: 13). Por último, en el plano funcional y teleológico, el problema reside en confundir los principios éticos con los jurídicos, cuando los primeros, a diferencia de los segundos, no son obligatorios o vinculantes por naturaleza de manera que no hay garantías sobre su observancia y respeto más allá del compromiso voluntario de cada sujeto. En la doctrina, Castel y Castel y Wagner, entre muchos otros, han advertido con sólidos argumentos sobre esta inquietante tendencia.

Los principios éticos no son obligatorios, ni pueden imponerse. No son universales ni tampoco homogéneos. La ética y el derecho establecen modelos de comportamiento necesarios para la organización y el funcionamiento de la sociedad, pero son distintos en su alcance y naturaleza. Las normas jurídicas son obligatorias, cuentan con una legitimidad propia y su cumplimiento se encuentra garantizado jurídica y judicialmente ${ }^{1}$. Las diferencias son evidentes y cada disciplina cumple su función.

c) El discurso jurídico sobre la IA resulta controvertido, básicamente, por dos motivos: 1) La importancia otorgada a la perspectiva ética, infravalorando o soslayando la necesidad de normas jurídicas vinculantes; y 2) La afirmación de la existencia de un vacío legal (Scherer, 2016: 356). La necesidad y la importancia de regular la IA cuentan, sin embargo, con un amplio apoyo institucional y doctrinal (Castel y Castel: 2016). Como advierte Maas, jurídicamente, "the rise of AI is clearly set to lead to urgent new questions for global governance" (Maas, 2019: 5). Burri reconoce que "the numerous processes currently under way are creating

\footnotetext{
${ }^{1}$ Un valor como la dignidad humana constituye simultáneamente un principio ético y un derecho. Desde la perspectiva ética, la dignidad puede ser objeto de diferentes interpretaciones como consecuencia de las concepciones y tradicionales culturales, ideológicas o religiosas y, en todo caso, carece de mecanismos para garantizar su respeto porque traduce un compromiso voluntario del sujeto con la idea. Desde la perspectiva jurídica, la dignidad es un derecho reconocido internacional y constitucionalmente con un alcance y un significado precisos y con mecanismos de garantía de su cumplimiento.
} 
international standards (...). The standards will be a kind of supersoft law .... they will be persuasive on their merits and imbued with a strong compliance pull, despite their non-binding form" (Burri, 2017: 106). Siguiendo a Boutin, "addressing responsibility and other regulatory and governance challenges is essential to advance the promises of new technologies, and international law can provide a platform for this endeavor" (Boutin, 2018: 7). En su opinion, en particular, "International law and international institutions can help addressing these challenges in a number of ways, by (1) coordinating the development of private standards, (2) adapting existing norms and concepts and filling regulatory gaps, and (3) providing frameworks for responsibility" (Boutin, 2018: 5). El análisis de la práctica pone de manifiesto que, tanto a nivel interno como internacional, hay que abordar la definición de la gobernanza de la IA.

\subsection{La Situación Práctica}

En un estudio publicado en julio de 2019 se advierte que solo 41 de los 193 Estados miembros de Naciones Unidas está trabajando en la preparación de medidas de gobernanza de la IA. Solo 19 han adoptado una estrategia o un plan nacional. Estados Unidos, China y la Unión Europea (UE) protagonizan el desarrollo de la IA a nivel mundial, pero cada uno traslada un modelo propio de desarrollo y comprensión de la misma. El rédito económico, el control de los ciudadanos y la visión humano-céntrica son, respectivamente, los parámetros que definen cada uno de aquellos modelos (López de Mántaras, 2019). Las estrategias nacionales no responden a patrones comunes, mientras que la acción en el plano internacional se caracteriza por la presencia de una multiplicidad de organizaciones y foros de discusión (a) y la prevalencia de estructuras de cooperación con una representación mayoritaria de países desarrollados (b).

a) La IA está siendo considerada en una pluralidad de organizaciones y foros regionales e interregionales, como corresponde a su importancia. Pero, con frecuencia, se producen solapamientos o duplicación del trabajo, siendo esta una situación poco recomendable en términos de economía y transparencia de los procesos. El caso de la UE es emblemático. En abril de 2018, la UE y sus Estados miembros aprueban una Declaración sobre cooperación en Inteligencia Artificial. En diciembre, la Comisión y los Estados miembros publican el Plan Coordinado Europeo sobre la Inteligencia Artificial. En abril de 2019, la Comisión adopta la Comunicación "Generar confianza en la inteligencia artificial centrada en el ser humano" y las "Directrices éticas para una Inteligencia Artificial Confiable". La sucesión de actos podría estar justificada. Pero no se explica la acumulación de estructuras y foros de discusión: el Grupo Europeo de Ética en Ciencia y Nuevas Tecnologías; el proyecto AI4People; el Grupo de Expertos de Alto Nivel sobre Inteligencia Artificial; y la Alianza Europea para IA.

b) Además de la UE y el Consejo de Europa, la OCDE, el G-7 y el G-20 están centralizando el debate sobre la gobernanza de la IA. El 22 de mayo de 2019, el Consejo de la OCDE adopta la Recomendación sobre la Inteligencia Artificial que es un documento, jurídicamente no vinculante, que marca las directrices que han acordado los Estados como principios rectores de la IA y para la cooperación internacional y el desarrollo de políticas internas. El 9 de junio de 2019, en la reunión ministerial celebrada en Tsukuba, el G-20 apoya esta recomendación. El G-7 adopta en junio de 2018 la "Charlevoix common vision for the future of artificial intelligence" que, con una estructura diferente, marca prácticamente los contenidos de la Recomendación adoptada por la OCDE. Los trabajos se desarrollan, en consecuencia, en un marco regional o interregional del que están ausentes los países menos desarrollados cuando, por la entidad del tema, habría de ser un debate universal.

En el contexto general de Naciones Unidas, no se ha articulado una respuesta global al tema de la gobernanza de la IA, sino que se está operando en ámbitos concretos con un grado de desarrollo desigual. En el informe "A United Nations system-wide strategic approach and road map for supporting capacity development on artificial intelligence" se propone un modelo de gobernanza multi-stakeholder y la atribución del liderazgo a la UIT como marco institucional centralizador de la actividad en esta materia. El Grupo de Alto Nivel sobre Cooperación Digital ha presentado una interesante propuesta de gobernanza ubicada dentro del sistema de Naciones Unidas que debe ser "inclusive (of multiple stakeholders), anticipatory (of fast-progressing AI technologies and impacts), responsive (to the rapidly evolving technology and its uses) and reflexive (critically reviews and updates its policy principles)". Esta propuesta defiende la aplicación de los principios de derecho internacional y plantea varias modalidades de gestión: una agencia especializada de la $\mathrm{ONU}$, una organización vinculada como la OMC o un órgano subsidiario de la Asamblea General. 
El estudio de la práctica pone de manifiesto que no hay un modelo de gobernanza a nivel universal, la mayoría de las iniciativas están protagonizadas por organizaciones regionales compuestas por países tecnológicamente desarrollados $\mathrm{y}$, en el plano nacional, son muy pocos los Estados que han elaborado propuestas sobre IA. Ante esta situación, de escasos y limitados avances y compromisos, hay quien ha afirmado la existencia de un vacío legal. No es cierto. Hay modalidades de respuesta jurídica para la IA.

\subsection{Metodología Jurídica de Respuesta}

La gobernanza de la IA es, posiblemente, uno de los mayores desafíos para la sociedad y el derecho. La IA no es una categoría estanca, sino permeable, transversal, porque actúa sobre los elementos y realidades preexistentes alterando su organización y funcionamiento. No es, por tanto, comprensible desde modelos convencionales de pensamiento, ni es posible proceder a su gestión y regulación desde parámetros tradicionales, previos, creados para una sociedad en la que no existía un desarrollo científico y tecnológico de este alcance y naturaleza.

Maas distingue tres tipos de respuesta jurídica a tecnologías disruptivas como la IA: "legal development", "legal displacement" and "legal destruction" (Maas, 2019: 2-4). La inviabilidad de las dos últimas opciones, acreditada jurídica y doctrinalmente, deja como única alternativa el primer nivel de análisis: el desarrollo jurídico. Esta metodología es válida en las diferentes situaciones que plantea la regulación de la IA. Moses explica, además, los cuatro supuestos que justifican el cambio jurídico como respuesta al cambio tecnológico, a saber: 1) La necesidad de crear nuevas normas específicas para regular, gestionar o prohibir determinadas técnicas o aplicaciones; 2) La incertidumbre jurídica sobre la manera de aplicarles el derecho en vigor; 3) El alcance incorrecto de la normativa porque fue creada con anterioridad de manera que puede incluir o excluir inadecuadamente conductas o situaciones; y 4) La obsolescencia jurídica porque, en ese nuevo contexto, la norma no es necesaria o no está justificada (Moses, 2007: 594-595).

En cualquier caso, con independencia del necesario desarrollo jurídico, no hay un vacío legal por varios motivos. En primer lugar, en Derecho internacional, hay principios y normas de naturaleza imperativa aplicables a cualquier ámbito de actividad. Dos ejemplos diferentes son la prohibición del uso o de la amenaza de la fuerza armada y la protección de los derechos y libertades de la persona. En segundo lugar, hay normas obligatorias asumidas por los Estados que puedan aplicarse por analogía a la IA. La protección de consumidores y las reglas en materia de responsabilidad serían extrapolables a este ámbito. En tercer lugar, hay normas que han de revisarse, pero no pueden cuestionarse, cuando se trata de aplicarlas en esta materia. Sería el caso de la protección de datos personales. Para terminar, habría que formular nuevas normas que respondan a los problemas específicos de este fenómeno como el principio de control humano o el estatuto jurídico de la IA.

\section{CONCLUSIÓN}

La gobernanza de la IA es difícilmente gestionable si no existe un concepto consensuado y aceptado, formulado jurídicamente, para dotarlo de mayor seguridad y garantías, sobre el que operar para garantizar la viabilidad del debate. Mientras que no exista acuerdo sobre el concepto difícilmente se podrá avanzar de un modo constructivo. Sobre la base de ese concepto, sería posible distinguir los dispositivos, programas o artificios que pueden ser o no calificados como IA y se podría, asimismo, establecer diferentes categorías que puedan requerir un tratamiento específico. En la conceptuación y categorización de la IA es fundamental adoptar una metodología interdisciplinar en la que, principalmente, la tecnología, la ética y el derecho contribuyan a establecer el modelo de gobernanza. El debate no puede quedar circunscrito a los técnicos, ni tampoco puede reducirse al establecimiento de principios éticos. La técnica es un medio y no un fin. La ética es un modelo de comportamiento voluntario, no universal, ni uniforme, que no puede ni, legítimamente, debe imponerse. El derecho es un instrumento de ordenación social que cuenta con mecanismos jurídicos y jurisdiccionales para garantizar su cumplimiento y cuenta, sobre todo, con el marco de legitimidad que ofrecen los procesos y sistemas normativos.

La IA requiere una regulación acorde a sus características y a su singularidad como objeto de conocimiento. El análisis de la práctica pone de manifiesto que, a nivel nacional, la mayoría de los Estados aún no ha desarrollado estrategias o planes de gobernanza. En el plano internacional, la situación tampoco es 
satisfactoria. En el marco universal, la acción de Naciones Unidas es limitada. Mientras, las iniciativas desarrolladas a nivel regional o interregional evidencian una situación caracterizada por la pluralidad y diversidad de foros de debate y, lo que es más grave, por la monopolización del mismo por parte de los países más desarrollados. Esta situación no implica que exista un vacío jurídico. Es cierto que hay que realizar cambios jurídicos para afrontar el cambio tecnológico que implica la IA, pero asumiendo la fórmula del "Legal Development".

Desde ese planteamiento, la ONU habría de asumir un rol principal, en particular, para evitar la sectorialización o la monopolización del debate por parte de los países más desarrollados. La aplicación de los principios de Derecho internacional de carácter imperativo al ámbito de la IA es, por su propia naturaleza, difícilmente cuestionable. Ello es válido, en particular, para materias como la seguridad internacional y la protección de los derechos y libertades fundamentales. Las normas obligatorias de carácter dispositivo también serían aplicables, directa o analógicamente, aunque pueden exigir adaptaciones para ajustarse a las características singulares de la IA. La creación ex novo de normas específicas para la IA será necesaria siempre y cuando aparezcan nuevas necesidades regulatorias o resulten insuficientes las normativas prexistentes.

La IA plantea tres grandes desafíos estructurales: un desafío social en la medida en que afecta a los derechos y libertades fundamentales del ser humano como tal y como componente de la sociedad; un desafío político, en la medida en que afecta a la organización y al funcionamiento del Estado de Derecho; y un desafío económico en cuanto altera los parámetros de desarrollo de los modelos económicos contribuyendo negativamente al aumento de las diferencias de desarrollo y al incremento de la denominada brecha digital. La respuesta a esos desafíos ha de ser la función esencial del marco de gobernanza de la IA.

\section{AGRADECIMIENTO}

Este trabajo ha sido financiado parcialmente por el Gobierno de España, con fondos FEDER, a través del Proyecto TIN2017-83494-R.

\section{REFERENCIAS}

Benvenisti, E., 2018. Upholding Democracy Amid the Challenges of New Technology: What Role for the Law of Global Governance. The European Journal of International Law, Vol. 29, No. 1, pp. 56 y ss.

Boutin, B., 2018. Technologies for International Law \& International Law for Technologies. In Groningen Journal of International Law, Vol. 6, pp. 1-8.

Burri, T., 2017. International Law and Artificial Intelligence. In German Yearbook of International Law, Vol. 60, pp. $91-108$.

Castel, J.-G. and Castel, M.E., 2016. The Road to Artificial Super-intelligence: Has International Law a Role to Play?. In Canadian Journal of Law and Technology, Vol. 14, No. 1, pp. 1-15.

Danaher, J., 2016. "The Threat of Algocracy: Reality, Resistance and Accommodation”. In Philosophy and Technology, Vol. 29, No. 3, pp. 245-268.

DeNardis, L. y Musiani, F., 2014. Governance by Infrastructure. Disponible en: https://ssrn.com/abstract=2730689 or http://dx.doi.org/10.2139/ssrn.2730689.

Habermas, J., 1986. Ciencia y técnica como “ideología”. Tecnos. Barcelona.

López de Mantaras, R., 2019. Inteligencia artificial, robotización y cuarta revolución digital. In Diálogos sobre democracias bajo control, 8, Fundación Ernest Lluch, Ed. Milenio, Barcelona, 2019, pp. 45-70.

Maas, M.M., 2019. International Law Does Not Compute: Artificial Intelligence and the Development, Displacement or Destruction of the Global Legal Order. In Melbourne Journal of International Law, Vol. 20, pp. 1-29.

Moses, L.B., 2007. Why Have a Theory of Law and Technological Change? In Minn. J.L. Sci. \& Tech., Vol. 8, pp. 589-606.

of diplomacy. Disponible en: https://www.diplomacy.edu/AI-diplo-report.

Scherer, M.U., 2017. Regulating Artificial Intelligence Systems: Risks, Challenges, Competencies, and Strategies. In Harvard Journal of Law \& Technology, Vol 29, No. 2, pp. 354-400.

Wagner, B., 2018. Ethics as an Escape from Regulation: From ethics-washing to ethics-shopping?. In M. Hildebrandt (Ed.). Being Profiling. Cogitas ergo sum. Amsterdam University Press. Ámsterdam. 